\title{
Innate immune detection of lipid oxidation as a threat assessment strategy
}

\section{Dania Zhivaki and Jonathan C. Kagan(1)}

Abstract $\mid$ Oxidized phospholipids that result from tissue injury operate as immunomodulatory signals that, depending on the context, lead to proinflammatory or anti-inflammatory responses. In this Perspective, we posit that cells of the innate immune system use the presence of oxidized lipids as a generic indicator of threat to the host. Similarly to how pathogen-associated molecular patterns represent general indicators of microbial encounters, oxidized lipids may be the most common molecular feature of an injured tissue. Therefore, microbial detection in the absence of oxidized lipids may indicate encounters with avirulent microorganisms. By contrast, microbial detection and detection of oxidized lipids would indicate encounters with replicating microorganisms, thereby inducing a heightened inflammatory and defensive response. Here we review recent studies supporting this idea. We focus on the biology of oxidized phosphocholines, which have emerged as context-dependent regulators of immunity. We highlight emerging functions of oxidized phosphocholines in dendritic cells and macrophages that drive unique inflammasome and migratory activities and hypermetabolic states. We describe how these lipids hyperactivate dendritic cells to stimulate antitumour $\mathrm{CD} 8^{+} \mathrm{T}$ cell immunity and discuss the potential implications of the newly described activities of oxidized phosphocholines in host defence.

The innate and adaptive arms of immunity operate as generalists and specialists, respectively. General molecular patterns that are associated with microbial life are used by pattern recognition receptors (PRRs) to detect potential infections ${ }^{1,2}$ (BOX 1). The microbial molecules are referred to as pathogen-associated molecular patterns (PAMPs). However, the specific identity of the potential pathogen is not identified by PRRs ${ }^{1}$. This specificity is provided by lymphocytes that bear antigen receptors, which are precise in their identification of individual pathogens. The link between generalists (PRRs) and specialists (antigen receptors) is causal, as genetic evidence indicates that PRR signalling on dendritic cells (DCs) is critical to stimulate antigen-specific responses by $\mathrm{T}$ cells $\mathrm{s}^{3-6}$.

While PRRs are not designed to identify specific microorganisms, increasing evidence indicates that innate immune networks operate as threat-assessment stations, which can distinguish between microbial and pathogenic encounters? ${ }^{7}$. The most commonly discussed means of distinguishing microorganisms from pathogens is through the coincident detection of PAMPs and virulence factor activities by distinct innate immune receptors $^{8,9}$. Microorganisms contain PAMPs but have no virulence factors, whereas pathogens contain both ${ }^{9}$. An example of threat assessment involves the induction of pyroptosis, an inflammatory form of cell death that can be triggered upon coincident detection of PAMPs and virulence factor activities $^{10-13}$. One of the challenges of this strategy of threat assessment is the unique repertoire of virulence factors encoded by individual pathogens. A general means of identifying threats to the host, regardless of the virulence factor repertoire, may be useful and more symmetrical to the PAMP-based strategy of microbial detection used by PRRs.
Herein we discuss data suggesting a distinct strategy of threat assessment by the innate immune system, which is not mediated by specific virulence factors. This process is rather mediated by one of the most common consequences of pathogen replication - oxidation of phospholipids in the environment following tissue injury. We discuss how oxidized phospholipids (oxPLs) operate as immunomodulatory signals that may provide a generic indication of the context of PAMP detection to the host. PAMP detection in the absence of oxPLs may represent contexts where non-replicating microorganisms have been encountered (that is, low threat encounters). By contrast, PAMP detection in the presence of oxidized products may represent contexts of high threat, where replicating microorganisms (that is, pathogens) have been encountered. The benefit of such a strategy is that tissue injury-induced oxidation may be considered a common (and perhaps unavoidable) outcome of pathogen replication, regardless of the virulence factor repertoire. Thus, akin to the generic use of PAMPs as an indicator of microorganisms, the innate immune system may use PAMPs plus oxPLs as generic indicators of heightened threat. The mechanisms and consequences of oxPL detection by the innate immune system are discussed herein.

\section{Phospholipid oxidation and recognition} Phospholipids such as phosphocholines are major constituents of mammalian cells. Under conditions of non-inflammatory or inflammatory tissue injury, any cell death that occurs in the local environment will result in the generation of reactive oxygen species (ROS). The source of the ROS may result from the actions of infiltrating neutrophils, dying mitochondria or the air itself. Under any of these conditions, phosphocholines in the plasma membrane can be oxidized. Defined examples of oxPL generation include tissue injury, lung infection in which an initial encounter with a pathogen is followed by tissue damage ${ }^{14}$, and during chronic inflammatory diseases such as atherosclerosis ${ }^{15}$. Mechanisms of oxPL generation are summarized in BOX 2 . In recent years, much focus has been on the arachidonic acid-containing phospholipid 


\section{Box 1 | Pattern recognition receptors sensing PAMPs and DAMPs}

Mammalian cells detect detrimental changes to the host such as potentially pathogenic microorganisms or host-encoded molecules indicative of tissue injury. This threat surveillance is achieved via the actions of a superfamily of pattern recognition receptors (PRRs), which recognize conserved ligands defined by classical terms as pathogen-associated molecular patterns (PAMPs) or damage-associated molecular patterns (DAMPs) ${ }^{2,68,69}$. Some of the most inflammatory PAMPs include lipopolysaccharides (LPS), outer-membrane components of Gram-negative bacteria. Upon bacterial encounters, LPS-binding protein (LBP) and CD14 operate in tandem to extract individual LPS molecules from bacterial cell walls and deliver these lipids to membrane-associated MD2 and Toll-like receptor 4 (TLR4). LPS-bound CD14 also induces an inflammatory endocytosis pathway that delivers TLR4 to endosomes ${ }^{22}$. This interaction activates inflammatory gene expression through nuclear factor- $\mathrm{kB}$ and mitogen-activated protein kinase signalling. Other LPS-interacting proteins have been described in detail in recent reviews ${ }^{13,70}$. Recently, it was shown that mammalian PRRs are unable to detect the LPS of most bacteria from a different ecosystem, such as deep sea bacteria, despite retaining most structural features of Escherichia coli LPS $^{71}$. These data suggest that pattern recognition of structurally conserved ligands may be defined locally, not globally. In addition to detecting microbial products, several PRRs recognize DAMPs, such as oxidized 1-palmitoyl-2-arachidonoyl-sn-glycero-3-phosphocholine derivatives (oxPAPCs) ${ }^{72}$. As opposed to our increasing understanding of how PAMPs and virulence factors are recognized, numerous questions remain unanswered regarding DAMP detection and signalling. 1-palmitoyl-2-arachidonoyl-sn-glycero-

3-phosphocholine (PAPC), which is a constituent of membranes in mammalian cells. Unlike their non-oxidized counterparts, oxPLs serve as damageassociated molecular patterns (DAMPs) and can modulate inflammation. Upon exposure to ROS, PAPC is oxidized at various positions to create a heterogeneous mixture of lipids collectively referred to as oxPAPCs. oxPAPCs are composed of a mixture of full-chain and truncated oxidized phosphocholine derivatives, which can be detected on dying cells and approach millimolar concentration in damaged tissues ${ }^{16}$. Growing interest has been focused on two components of oxPAPC namely, 1-palmitoyl-2-glutaroyl-snglycero-3-phosphocholine (PGPC) and 1-palmitoyl-2-(5-oxovaleroyl)-sn-glycero3-phosphocholine (POVPC) ${ }^{17}$. As opposed to PAMPs, which are intrinsically inflammatory, it is impossible to describe oxPAPCs as inducers or inhibitors of inflammation in all contexts. Rather, oxPAPCs display context-dependent activities, which is likely to be an important aspect of threat assessment.

Several oxPAPC receptors have been defined, each of which may impact oxPAPC-mediated inflammatory activity in different ways. On macrophages, the scavenger receptor CD36 binds oxPAPCs and induces their uptake. This process promotes foam cell formation during atherosclerosis ${ }^{18}$. The structural basis of oxPAPC binding to CD36 has been defined, with Lys164 and Lys 166 being required for the binding with $s n-2$ acyl chains of oxPAPC ${ }^{19}$. oxPAPC binding to CD36 is blocked by E06, a monoclonal IgM antibody that recognizes the phosphocholine headgroup of oxPAPC (see BOX 3), demonstrating the importance of the oxPAPC headgroup in binding to CD36 $\left(\right.$ REF. $^{20}$ ).

In addition to CD36, oxPAPCs bind the bacterial lipopolysaccharide (LPS) receptor
CD14 on myeloid cells, thereby allowing oxPAPC internalization into endosomes and transport to the cell cytosol ${ }^{21}$. In the context of oxPAPC-induced inflammatory activities (described in detail later), CD14 serves as the primary receptor for these oxPLs ${ }^{21}$. CD14 functions as a transporter associated with the execution of inflammation (TAXI) protein $^{22}$, which delivers LPS from the bacterial cell wall to membrane-associated MD2 and Toll-like receptor 4 (TLR4). CD14 then transports LPS and TLR4 to signalling-competent subcellular regions $^{22}$. Similarly to LPS, the interaction between oxPAPCs and CD14 results in CD14 endocytosis and depletion of CD14 from the plasma membrane ${ }^{21}$. Therefore, oxPAPCs compete with LPS for CD14 binding in cell-free experimental systems, an activity that likely explains why the pretreatment of naive cells with oxPAPCs blocks subsequent responses to LPS via TLR4 (REF. ${ }^{23}$ ).

Upon CD14-dependent endocytosis in macrophages and DCs, oxPAPCs gain access to the cytosol, where they bind the inflammasome regulators caspase 1

\section{Box 2 | Mechanisms of oxidized phosphocholine generation and physiological accumulation}

Phospholipids such as the arachidonic acid-containing phospholipid 1-palmitoyl-2-arachidonoylsn-glycero-3-phosphocholine (PAPC) are essential components of the plasma membrane of every cell type ${ }^{73}$, lung surfactant ${ }^{74}$ and circulating lipoproteins ${ }^{75}$. PAPCs are composed of fatty acids bound to a glycerol backbone containing a polar head group. The second position, also known as the sn-2 chain, in the glycerol backbone contains esterified monounsaturated or polyunsaturated fatty acids that are prone to oxidation by free radicals or enzymatically by myeloperoxidase or lipoxygenase. PAPCs react with oxygen to create a mixture of oxidized phospholipids, collectedly referred to as oxPAPCs. Enzymatically oxidized phospholipids are key signalling mediators that regulate various cellular and physiological processes, including thrombosis, metabolism and vascular inflammation ${ }^{76}$. Accordingly, oxidized lipids were shown to modulate responses by innate immune cells, including neutrophils, platelets and peritoneal macrophages ${ }^{62}$. A detailed description of the mechanism of generation of enzymatically oxidized lipids as well as their regulatory role in innate immunity is provided elsewhere ${ }^{77}$.

Physiologically, the pool of oxPAPCs can also be generated non-enzymatically from cellular membranes, by reactive oxygen and nitrogen species from endogenous sources (for example, mitochondrial respiratory chain and NADPH oxidase) or exogenous sources (air pollution, smoking and UVB light $)^{30}$. In the absence of pathogenic infections, oxPAPCs accumulate in dying cells, in membrane vesicles released from activated cells $s^{78,79}$ and in oxidized low-density lipoproteins ${ }^{80}$ and oxidized pulmonary surfactant ${ }^{14}$. Furthermore, diverse inducers of lung inflammation, such as influenza viruses ( $\mathrm{H} 5 \mathrm{~N} 1, \mathrm{H} 1 \mathrm{~N} 1$ and $\mathrm{H} 3 \mathrm{~N} 2$ ), monkeypox virus, Yersinia pestis, Bacillus anthracis and severe acute respiratory syndrome coronavirus, can increase the levels of oxPAPCs ${ }^{14}$. Studies demonstrating evidence of oxPAPC accumulation in different infectious diseases, ranging from mycobacterial infections to sepsis and during various respiratory viral infections, have recently been reviewed ${ }^{29}$. In addition, oxPAPCs are generated in the cerebrospinal fluid of individuals with multiple sclerosis ${ }^{81,82}$, suggesting that oxPAPCs may contribute to the pathogenesis of autoimmune demyelination. The level of bioactive 1-palmitoyl-2-glutaroyl-sn-glycero-3-phosphocholine was found to be elevated in a broad range of cardiovascular injuries, such as atherosclerosis and ischaemia-reperfusion injury, during age-related macular degeneration in humans and in patients with alcoholic liver inflammation ${ }^{30}$ and non-alcoholic steatohepatitis ${ }^{83}$. There is a growing interest in using oxPAPCs as biomarkers of human diseases, particularly for cardiovascular diseases ${ }^{84}$. Increasing evidence indicates that individual moieties contained in oxPAPCs exert non-redundant proinflammatory effects in macrophages and dendritic cells. Further work is needed to better understand the scope of threats to the host where these bioactive oxidized phospholipids could arise and their influence on immune responses and diseases. 


\section{Box 3 | Natural antibody-mediated recognition of oxidized phosphocholines}

Oxidized phospholipids (oxPLs) released from dying cells serve as antigens for natural antibodies. Therefore, antibody-based enzyme-linked immunosorbent assays are used as a method to detect and quantify oxPLs. Three monoclonal antibodies are specific for oxPLs (E06, DLH3 and 509) ${ }^{30}$. E06 and DLH3 are specific for phosphatidylcholine moieties, whereas 509 specifically recognizes oxidized phosphatidylethanolamine ${ }^{85}$. E06 is the best characterized oxPL-specific antibody, and was cloned from hybridomas generated from APOE-deficient mice and is commonly used to quantify oxidized 1-palmitoyl-2-arachidonoyl-sn-glycero-3-phosphocholine derivatives (oxPAPCs). However, E06 cannot distinguish between the distinct molecular species of oxPAPCs such as 1-palmitoyl-2-glutaroyl-sn-glycero-3-phosphocholine, 1-palmitoyl-2-(5-oxovaleroyl)sn-glycero-3-phosphocholine (and 1-palmitoyl-2-(5,6-epoxyisoprostane $\mathrm{E}_{2}$ )-sn-glycero3-phosphocholine. Such differentiation is important because available data show that individual molecular species often have different biological activities. Imai et al. used E06 to quantify the levels of oxPAPCs in murine lung injuries caused by H5N1 avian influenza virus and acid instillation ${ }^{14}$. Furthermore, using E06 antibody, this group showed that oxPAPCs accumulate during infections in the lungs of humans and animals infected with anthrax or severe acute respiratory syndrome coronavirus. The accumulation of E06-reactive oxPAPCs was also detected in dermal fibroblasts upon skin exposure to UV radiation ${ }^{86}$. It is speculated that E06 may be useful for therapeutic targeting of inflamed tissues in various inflammatory diseases. As a proof of concept, within mice, E06 activities in mice reduced inflammation and attenuated the progression of atherosclerosis, aortic stenosis and hepatic steatosis ${ }^{87}$. In addition, treatment with the monoclonal $\lg \mathrm{G} 1$ antibody X19-mu, which exhibits properties similar to those of the endogenous IgM E06 antibody, preserved coronary function and attenuated atherosclerosis in mice ${ }^{88}$. The presence of natural oxPL-reactive antibodies was detected during inflammation using an anti-idiotypic antibody that specifically recognizes the idiotope of E06, and oxPAPC-reactive antibodies were found in plaques of patients with multiple sclerosis and in mouse models ${ }^{81}$. The role of these anti-oxPL antibodies is still unknown and requires further evaluation.

and caspase $11\left(\mathrm{REF}^{24}\right)$. oxPAPC-caspase interactions are thought to stimulate NLR family pyrin domain-containing protein 3 (NLRP3) inflammasome assembly in a potassium-independent manner ${ }^{24}$. Like CD14, caspase 11 interacts with oxPAPCs and LPS. In vitro studies demonstrated that LPS interacts with the caspase activation and recruitment domain (CARD) of caspase 11, whereas oxPAPCs bind the catalytic domain of caspase $11\left(\mathrm{REF}^{24}\right)$. As a consequence, the functional impacts of oxPAPCs and LPS on caspase 11 are distinct. When LPS binds to caspase 11 in macrophages or DCs, this interaction induces inflammasome-

dependent IL- $1 \beta$ release from macrophages, which is associated with pyroptosis.

By contrast, oxPAPC binding to caspase 11 induces the release of IL- $1 \beta$ from living DCs, in the absence of pyroptosis ${ }^{24}$. The ability of oxPAPCs to bind the catalytic domain of caspase 11 was not associated with stimulation of its enzymatic activity, as is typically observed when inflammatory LPS structures interact with the caspase 11 CARD $^{25,26}$. Rather, oxPAPCs prevent intrinsic and LPS-induced caspase 11 activity $^{24}$. This finding, coupled with the aforementioned competition between oxPAPCs and LPS for CD14, may explain why LPS-induced inflammation can be prevented by pretreatment of cells or mice with oxPAPCs. Many questions remain unanswered with regard to the biochemical interaction of oxPAPCs with CD14 and inflammatory caspases. In addition, how CD14 delivers oxPAPCs to the cytosol remains ambiguous, but it is possible that oxPAPCs alter the endosomal membrane and provoke their own leakage into the cytosol $^{27}$.

In summary, oxPAPCs are recognized by multiple receptors on myeloid cells, which may lead to the crosstalk of diverse signalling pathways and the modulation of myeloid cell functions that we further describe in the following sections.

\section{Consequences of oxPAPC recognition}

The detection of oxPAPCs by macrophages or DCs can impact inflammatory activities in a diverse range of diseases, including acute inflammation, atherosclerosis, cancer, lung injury and age-related diseases ${ }^{16,28,29}$.

Depending on the context in which oxPAPCs are encountered, these lipids exert proinflammatory or anti-inflammatory effects $^{30}$. For example, during conditions representative of sterile tissue injury, oxPAPCs exert anti-inflammatory functions, perhaps by preventing efficient detection of other molecules that stimulate CD14, TLR 4 or caspase 11 . By contrast, at the site of infection where tissue damage is common, oxPAPCs exhibit proinflammatory activities in cells that have also detected PAMPs. In the following subsections we describe how oxPAPCs regulate innate immune functions of myeloid cells and influence the induction of adaptive immunity.
Impact of oxPAPCs on phagocytosis and endocytosis. Accumulating evidence demonstrates that oxPAPCs modulate phagocytosis by antigen-presenting cells in a cell typespecific and context-specific manner. For example, oxPAPC pretreatment of peritoneal macrophages diminished their ability to internalize Escherichia coli. In mice, preexposure to oxPAPCs led to a reduction in host defence, resulting in bacterial outgrowth and systemic dissemination ${ }^{31}$. Similarly, intratracheal instillation of an oxPAPC mixture or pure phosphocholine moieties (for example, POVPC or PGPC) rendered mice unable to eradicate subsequent infections with Pseudomonas aeruginos $a^{32}$. oxPAPCs generated in the bronchoalveolar space of mice exposed to cigarette smoke impaired phagocytosis by alveolar macrophages $^{32}$. The link between these in vivo defects in phagocytosis after oxPAPC exposure was established by means of the monoclonal antibody E06, which binds to and prevents the activities of several oxPAPC component lipids. E06 treatment prevented the inhibition of bacterial phagocytosis and restored bacterial clearance ${ }^{32}$.

In contrast to the bacterial exposures described above, oxPLs can stimulate phagocytosis of apoptotic cells by macrophages via CD36 (REF. $\left.{ }^{33}\right)$. In the context of non-phagocytic cargo, oxPAPCs did not affect dextran or soluble ovalbumin uptake by endocytosis. Moreover, in the presence of oxPAPCs, the ability of LPS-primed DCs to cross-present peptides on MHC class I molecules was enhanced ${ }^{34}$. These data are in accordance with reports indicating that within DCs, antigen leakage from endosomes for cross-presentation may occur upon direct oxidation of endosomal lipids and generation of oxPLs ${ }^{27}$. Thus, depending on the cargo to be internalized, oxPAPCs exhibit contrasting effects on phagocytic and endocytic activities. The molecular basis for these context-dependent behaviours is undefined.

\section{Impact of oxPAPCs on inflammasome} activities. Following priming with various TLR ligands, oxPAPCs induce a long-lived state of hyperactivation in myeloid cells ${ }^{24}$. This activation state is distinct from naive, active or pyroptotic cell states. A description of these cell states is provided in BOX 4 and FIC. 1. A hallmark of oxPAPC-stimulated hyperactive cells is their ability to secrete inflammasome-dependent IL- $1 \beta$ while remaining viable. Extracellular oxPAPC components are detected by CD14 (REF. ${ }^{21}$ ). CD14-dependent endocytosis delivers these lipids to intracellular caspase 11. oxPAPC 
binding with caspase 11 leads to NLRP3dependent inflammasome assembly and IL- $1 \beta$ release in the absence of cell death ${ }^{24}$. In contrast to the rapid but transient burst of IL- $1 \beta$ release that occurs upon exposure of phagocytes to pyroptotic stimuli, oxPAPCs or PGPC promote IL- $1 \beta$ release from LPS-primed cells for several days ${ }^{21}$.

IL- $1 \beta$ is a highly inflammatory cytokine and its secretion is tightly regulated ${ }^{35}$. TLR signalling induces production of pro-IL- $1 \beta$, but this pro-protein form lacks an amino-terminal secretion signal and cannot be secreted via the endoplasmic reticulumGolgi network $^{35}$. To gain inflammatory activities, pro-IL- $1 \beta$ must be cleaved, most commonly by inflammatory caspases present within inflammasomes ${ }^{36}$. Inflammasomes are supramolecular organizing centres, which assemble in the cytoplasm following their activation ${ }^{36}$. Caspases that are activated within inflammasomes (most commonly caspase 1 ) cleave pro-IL- $1 \beta$ and gasdermin D (GSDMD). GSDMD cleavage unleashes the latent ability of its amino terminus to form pores in the plasma membrane. Pore formation is facilitated by interactions between GSDMD and acidic phosphoinositides present in the inner leaflet of the plasma membrane, such as phosphatidylinositol 4,5-bisphosphate and phosphatidylserine ${ }^{37}$. GSDMD pores serve as conduits for mature IL- $1 \beta$ secretion in a cell lysis-independent event ${ }^{38,39}$. Cargo transport across the negatively charged GSDMD conduit occurs via a size-exclusion mechanism ${ }^{38,39}$, as well as by electrostatic filtering of cytosolic proteins, which favours the release of the positively charged mature IL- $1 \beta$, while repelling pro-IL- $1 \beta^{40}$. If GSDMD pores are not repaired by the cell, pyroptotic cell death may occur, during which IL-1 $\beta$ and other cytosolic contents can be released non-selectively. Of the well-defined inflammasome agonists, including intracellular LPS, ATP, nigericin and alum, all induce pyroptosis. By contrast, within TLR ligand-stimulated cells, oxPAPCs induce inflammasome-dependent release of IL- $1 \beta$ through GSDMD pores in the absence of cell death. The ability of oxPAPCs to add IL- $1 \beta$ to the repertoire of factors that are normally secreted by living phagocytes renders these cells more immunostimulatory than their activated counterparts that have been stimulated only with PAMPs - hence the term 'hyperactive? Hyperactive cells display evidence of fewer GSDMD pores at their surface than their pyroptotic counterparts ${ }^{39}$. Whether GSDMD pores in hyperactive cells are regulated similarly as in pyroptotic cells downstream of inflammasome activation is unknown.

Hyperactive cells may undergo rapid membrane repair events that ensure viability. Indeed, pyroptotic events increase in cells that are defective for membrane repair mediated by the endosomal sorting complex required for transport machinery ${ }^{41}$.

Individual lipid moieties such as PGPC and POVPC, which constitute less than $10 \%$ of the total lipids present in the parental oxPAPC mixture ${ }^{21}$, induce NLRP3 inflammasome activation and the release of IL- $1 \beta$ from DCs and macrophages ${ }^{24}$. However, while the oxPAPC mixture induces a state of hyperactivation in DCs generated using granulocyte-macrophage colony-stimulating factor (GM-CSF), oxPAPCs were poor inducers of IL- $1 \beta$ secretion from the type 1 conventional DC (cDC1) subset or $\mathrm{CDC} 2$ subset of $\mathrm{DCs}^{34}$. In addition, oxPAPCs were capable of eliciting these responses only from DCs, not macrophages. Conversely, PGPC was able to induce inflammasome-dependent IL- $1 \beta$ secretion from living $\mathrm{cDC} 1 \mathrm{~s}, \mathrm{cDC} 2 \mathrm{~s}$ and macrophages ${ }^{34}$. All these oxPAPC components engage CD14 and promote inflammasome-mediated IL- $1 \beta$ release from living cells (FIG. 2). Much work remains to be done to define the molecular events that dictate the specific cell fate commitments induced by oxPAPCs or the component lipids. Additional activities of oxPAPC components have been identified, which are described in the following sections.

Impact of oxPAPCs on DC migratory
activity. Independently of their
inflammasome-stimulatory activities,
oxPAPCs promote the robust migratory
capacity of TLR-activated DCs. Therefore,
the hyperactivating stimuli LPS plus
oxPAPCs (or PGPC) exceeded other
activation stimuli (for example, LPS alone)

\section{Box 4 | Distinct activation states of dendritic cells and implication for immunotherapy}

In the absence of infection or damage, dendritic cells (DCs) exist in a non-inflammatory resting state. Upon detection of pathogen-associated molecular patterns or damage-associated molecular patterns, pattern recognition receptor activation unleashes signalling pathways that lead to a shift in DC activities from a naive state to an 'activated' state ${ }^{89,90}$. Activated DCs upregulate several factors, including co-stimulatory molecules (such as CD69 and CD80) and chemokine receptors (such as CC-chemokine receptor 7 (CCR7)) that are essential for their migration to adjacent lymphoid tissues to initiate an adaptive immune response ${ }^{4,91}$. During this activation process, polarizing cytokines are produced by DCs that determine the progressive differentiation of $\mathrm{CD} 4^{+} \mathrm{T}$ cells and $\mathrm{CD} 8^{+} T$ cells into effector cells and terminally differentiated memory cells. Active DCs have a life expectancy of only a few days, and are viewed as wellequipped antigen-presenting cells to stimulate de novo T cell priming ${ }^{92}$, and for boosting memory T cells. Therefore, several immunotherapies for cancer have been undertaken to use DCs to drive protective immunity ${ }^{93,94}$. These strategies often involve the use of microbial products that stimulate Toll-like receptors (TLRs), such as intratumoural administration of the TLR3 ligand poly(l:C) ${ }^{94}$. The clinical efficacy of $D C$ vaccines remains unsubstantiated. A potential reason for this clinical failure is that we lack a consensus on the optimal means to stimulate DC activities that drive protective immunity ${ }^{95,96}$. Among the polarizing cytokines that DCs need to produce is IL-1 $\beta$, a critical regulator of $\mathrm{T}$ cell differentiation, memory $\mathrm{T}$ cell generation and effector function ${ }^{53,97,98}$. However, TLR-stimulated DCs do not release this important cytokine, a finding that undermines the value of TLR-alone stimulations for cancer immunotherapy. DCs can be prompted to release IL-1 $\beta$ by treatments with chemical agonists of the inflammasome pathway, with the classic inflammasome agonists being those that stimulate an inflammatory form of cell death known as pyroptosis ${ }^{10,13,99,100}$. Pyroptotic DCs, however, cannot participate in the days-long process needed to prime and differentiate naive $T$ cells in the draining lymph node ${ }^{101}$. Accordingly, stimuli that promote pyroptosis, such as alum (the vaccine adjuvant approved by the US Food and Drug admin-

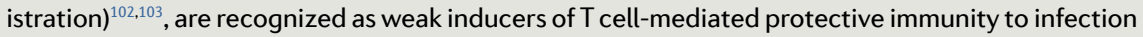
or cancer ${ }^{104}$. The ideal strategy of stimulating memory $T$ cell immunity is achieved by generating hyperactive $D C$ s, a recently identified activation state in which DCs have the ability to secrete inflammasome-dependent IL-1 $\beta$ while maintaining their viability ${ }^{24}$. When DCs are exposed to TLR ligands and oxidized 1-palmitoyl-2-arachidonoyl-sn-glycero-3-phosphocholine derivatives (oxPAPCs) or pure components of oxPAPCs (such as 1-palmitoyl-2-glutaroyl-sn-glycero3-phosphocholine or 1-palmitoyl-2-(5-oxovaleroyl)-sn-glycero-3-phosphocholine), DCs achieve a long-lived state of "hyperactivation" ${ }^{21,24}$. Notably, the type 1 conventional DC (cDC1) subset achieves a state of hyperactivation in vivo. Hyperactive $\mathrm{CDC} 1 \mathrm{~s}$ display common features with activated DCs (such as secretion of tumour necrosis factor), but they also gain the ability to simultaneously provide T cells with IL-1 $\beta$ over the course of several days ${ }^{21}$. Consequently, hyperactive $\mathrm{CDC} 1 \mathrm{~s}$ were identified as the most potent $\mathrm{DC}$ s in generating long-lived memory $\mathrm{CD} 8^{+}$ $T$ cells that eradicate tumours ${ }^{34}$. The identification of these distinct DC activation states provides a mandate to further explore the differential impact of hyperactive DCs compared with their naive, active or pyroptotic counterparts during immunopathogenesis. 


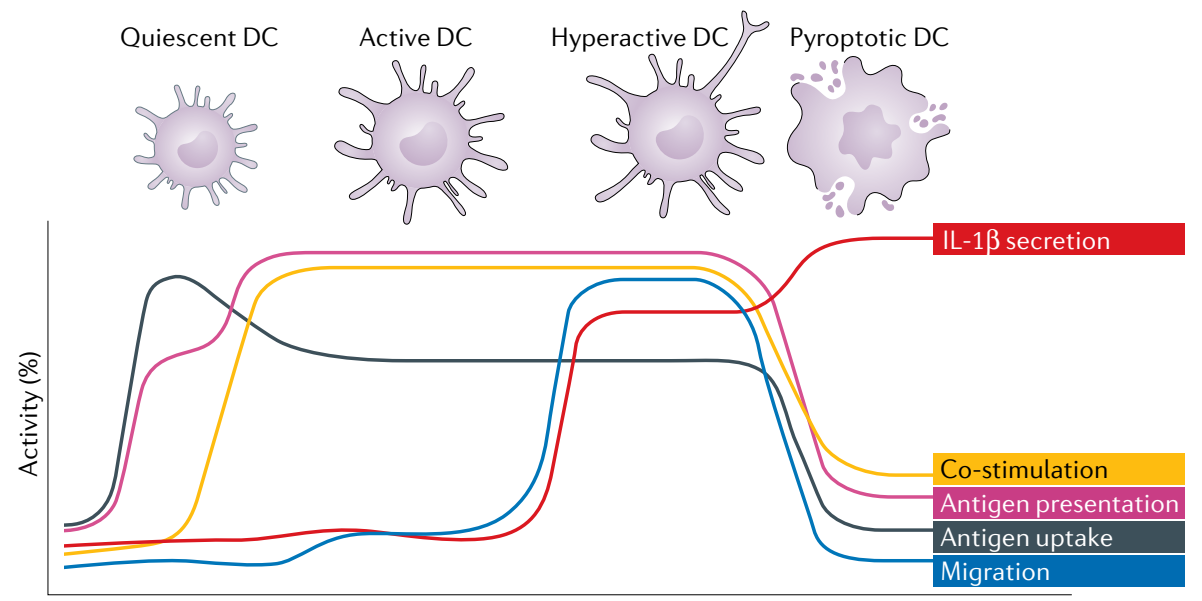

DC activation state

Fig. 1 | The T cell stimulatory activities of dendritic cells at distinct activation states. To regulate T cell activity, dendritic cells (DCs) provide T cells with several signals that are important for the establishment of an appropriate T cell response. Naive DCs are quiescent DCs that have the ability to take up antigens. Active DCs take up antigens and have an enhanced ability to present antigen peptides on MHC molecules. In addition, active DCs upregulate co-stimulatory molecules to stimulate T cells. Hyperactive DCs share similar activities with their active counterparts, but also gain the ability to hypermigrate to lymph nodes and to secrete IL-1 $\beta$. Pyroptotic DCs secrete high levels of IL-1 $\beta$. However, pyroptotic DCs are dead and lose their T cell stimulatory capacity.

in their ability to stimulate DC migration to adjacent draining lymph nodes ${ }^{34}$. RNA sequencing of the $\mathrm{CDC} 1$ and $\mathrm{CDC} 2$ subsets revealed that hyperactive DCs (primed with LPS and then stimulated with PGPC) upregulated the expression of several gene clusters involved in DC migration and chemotaxis ${ }^{34}$. Notably, DCs that were stimulated with LPS plus oxPAPCs or PGPC induced the upregulation of CCchemokine receptor 7 (CCR7). Accordingly, LPS plus oxPL treatment of DCs enhanced their migratory activities to the draining lymph nodes after adoptive transfer into the skin $^{34}$. The ability of oxPAPCs or their components to stimulate DC migration was independent of inflammasome activities, as no defects in DC migration were observed when studies were performed with cells lacking the inflammasome components NLRP 3 or caspases 1 and 11. Although these findings expand the scope of immunological activities mediated by oxPAPCs, the mechanism by which oxPAPCs or PGPC modulates DC motility is undefined.

\section{oxPAPC-mediated mitochondrial} reprogramming in macrophages. Within macrophages, oxPAPCs stimulate metabolic reprogramming, termed 'hypermetabolism' ${ }^{42}$. This metabolic state translates tissue oxidation status into heightened inflammatory gene expression. While macrophages activated by LPS downregulate their oxidative phosphorylation activities and rely on glycolysis for energy production, macrophages exposed to LPS and oxPAPCs used both oxidative phosphorylation and glycolysis. Under these conditions, the Krebs cycle is fed with glutamine, and pro-IL- $1 \beta$ production is enhanced by the increased activity of the transcription factor hypoxia-inducible factor $1 \alpha(H I F 1 \alpha)$. This process is mediated by the accumulation of the metabolite oxaloacetate in the cytoplasm ${ }^{42}$.

The significance of the hypermetabolic state induced by oxPAPCs was revealed in atherosclerosis models, such as $\mathrm{Ldlr}^{-1-}$ or $A_{p o e^{-/-}}$mice. When these mice were fed a high-fat diet, which drives oxPAPC accumulation in oxidized low-density lipoproteins, hypermetabolic phagocytes were identified in the blood. Interestingly, drugs that interfered with oxPAPC-driven metabolic changes reduced atherosclerotic plaque formation and protected mice against atherosclerosis development. It is speculated that this metabolic rewiring of macrophages increases mitochondrial fitness, which may prolong the lifespan of macrophages. In the context of infection, this hypermetabolic state may enhance host defence, but the same mechanism may underpin symptoms associated with heart disease and potentially other chronic inflammatory diseases.

Pure lipids contained within the oxPAPC mixture elicited distinct contributions to metabolic hyperinflammation. Whereas POVPC and PGPC drove inflammasome activation in macrophages, 1-palmitoyl-2-(5,6-epoxyisoprostane $\mathrm{E}_{2}$ )-sn-glycero-3-phosphocholine uniquely induced the hypermetabolic activities described above ${ }^{42}$. In another study, macrophages that were exposed to oxPAPCs acquired a unique redox-regulatory phenotype (referred to as Mox cells). Mox macrophages induce glutathione synthesis and depend on glucose to fuel cellular activities. This unusual macrophage phenotype was observed in atherosclerotic plaques of $\mathrm{Ldlr}^{-1-}$ mice. Additional details about the metabolic activities of oxPLs in phagocytes are described in a recent review $^{43}$.

\section{Impact of oxPAPC on immune responses oxPAPC-induced acute inflammatory}

responses. In the absence of PAMPs, oxPAPCs are weak inducers of cytokine production by myeloid cells ${ }^{24}$. In contrast to LPS, oxPAPCs did not induce TLR4 dimerization and endocytosis, myddosome formation or the expression of proinflammatory cytokines in macrophages $^{21}$. However, in the context of animals, in which exposures to microbial products are difficult to control, oxPAPCs exhibit proinflammatory responses via TLR4 $\left(\mathrm{REFS}^{14,43}\right)$. Upon lung injury, oxPAPCs promoted IL-6 secretion from alveolar macrophages, and played a critical role in lung disease pathogenesis via a TLR4-TICAM pathway. Neutralization of oxPAPCs by the antibody E06 attenuated IL-6 production in the bronchoalveolar fluid. Furthermore, Shirey et al. described strong inhibition of oxPAPC-induced IL-6 production after treatment with the TLR4 antagonist eritoran ${ }^{43}$. Thus, in airways, oxPAPCs are potent stimulators of inflammation.

In contrast to the activities of oxPAPCs in the lung, studies of the peritoneal cavity revealed that oxPAPCs prevented TLR4 signalling ${ }^{21}$ and were described as LPS antagonists $^{44,45}$. These anti-inflammatory activities of oxPAPCs in the peritoneum can be reproduced in vitro, where oxPAPCtreated phagocytes that were subsequently exposed to LPS exhibited defects in TLR4 dimerization and signalling. As a consequence, oxPAPC pretreatment inhibited LPS-inducible expression of interferonstimulated genes and secretion of proinflammatory cytokines ${ }^{21}$. In addition, several studies indicated that pretreatment of DCs or macrophages with oxPAPCs blocks nuclear factor- $\mathrm{KB}$ responses to subsequent LPS treatments ${ }^{46-49}$. Mechanistic studies revealed that this inhibition of TLR signalling occurred by competitive interaction of LPS or oxPAPCs with CD14 (REF. ${ }^{21}$ ). Indeed, oxPAPCs and LPS interact with 
CD14 using the identical amino acids, thus providing a biochemical explanation for their competitive activities ${ }^{21}$. These mechanistic findings likely explain why co-injection of oxPAPCs and LPS into mice protected mice from lethal sepsis ${ }^{46}$, as under these conditions, both of these lipids compete with one another for CD14.

Recent studies identified PECPC as the most potent anti-inflammatory oxPAPC component ${ }^{50}$. Additionally, a small synthetic analogue of PGPC named 'VB-201', in which the ester bonds at $s n-1$ and $s n-2$ of the glycerol backbone are replaced with ether bonds to enhance the molecule's stability against enzymatic degradation in vivo, was also shown to bind to CD14. VB-201 inhibits TLR2 and TLR4 in human and mouse monocytes and monocytederived DCs ${ }^{51}$. In addition, VB-201 administered orally ameliorated the severity of experimental autoimmune encephalomyelitis ${ }^{51}$, and inhibited liver inflammation in a mouse model of non-alcoholic steatohepatosis ${ }^{52}$.

These data highlight circumstances in which oxPAPCs are acutely inflammatory or immunosuppressive. These seemingly disparate observations may be explained by the mechanistic studies that have been performed. The proinflammatory activities of oxPAPCs in the lung may be explained by the presence of TLR4 ligands at this mucosal surface, which synergize with oxPAPCs to drive inflammation. By contrast, the anti-inflammatory activities in the peritoneal cavity may be explained by the lack of resident TLR ligands to synergize with oxPAPCs. In this latter context, oxPAPC injection leads to reduced inflammatory responses to subsequent bacterial infections, perhaps because oxPAPC pre-injection saturates cellular pools of CD14 that would normally be stimulated by bacterial products. Support for this idea was provided by recent data demonstrating that pretreatment of the peritoneal cavity of mice with LPS enabled oxPAPCs to induce inflammation $^{21}$. By contrast, oxPAPC injection into the peritoneum in the absence of LPS priming elicits no inflammatory response $^{21}$.

The immunomodulatory activities of oxPAPCs described above are associated with rapid innate immune responses that occur within minutes to hours of cellular exposure. In the next section we describe recent assessments of the impact of oxPAPCs on the long-term adaptive immune responses in the context of cancer.
oxPAPC-mediated control of adaptive immunity. oxPAPCs can regulate adaptive immunity by acting on antigen-presenting LPS and PGPC induced antitumour $\mathrm{CD}^{+}$ $T$ cell responses in mice that resulted from inflammasome activities within living DCs, and their hypermigratory ability that enables tumour antigens to reach lymphoid tissues (FIG. 3). These migratory DCs continuously secrete IL-1 $\beta$ to prime de novo T cells and activate memory $\mathrm{T}$ cells ${ }^{34,53}$. Consequently, LPS plus PGPC was a strong adjuvant to complex tumour antigens (for example, whole tumour lysates) during prophylactic or therapeutic immunizations against a range of murine tumours. Recent studies in humans revealed the need for multiple neoantigens cells. Hyperactive DCs stimulated with (up to 20 peptides) for effective generation of personalized cancer therapies ${ }^{54-56}$. The ability of oxPLs to adjuvant whole tumour lysates may bypass the challenge of neoantigen identification and enable immunotherapies to be developed that are agnostic to the identity of tumour antigens ${ }^{34}$. It is likely that hyperactivating stimuli uniquely elicit the generation of a wide diversity of antigen-specific T cells, perhaps due to their abundance in the draining lymph node. Indeed, oxPAPC-containing or PGPC-containing adjuvants potentiated the generation of antigen-specific $T$ cell responses and increased the kinetics of memory and effector $\mathrm{T}$ cell generation ${ }^{34}$.

In considering the underlying logic to the benefit of hyperactivating stimuli on

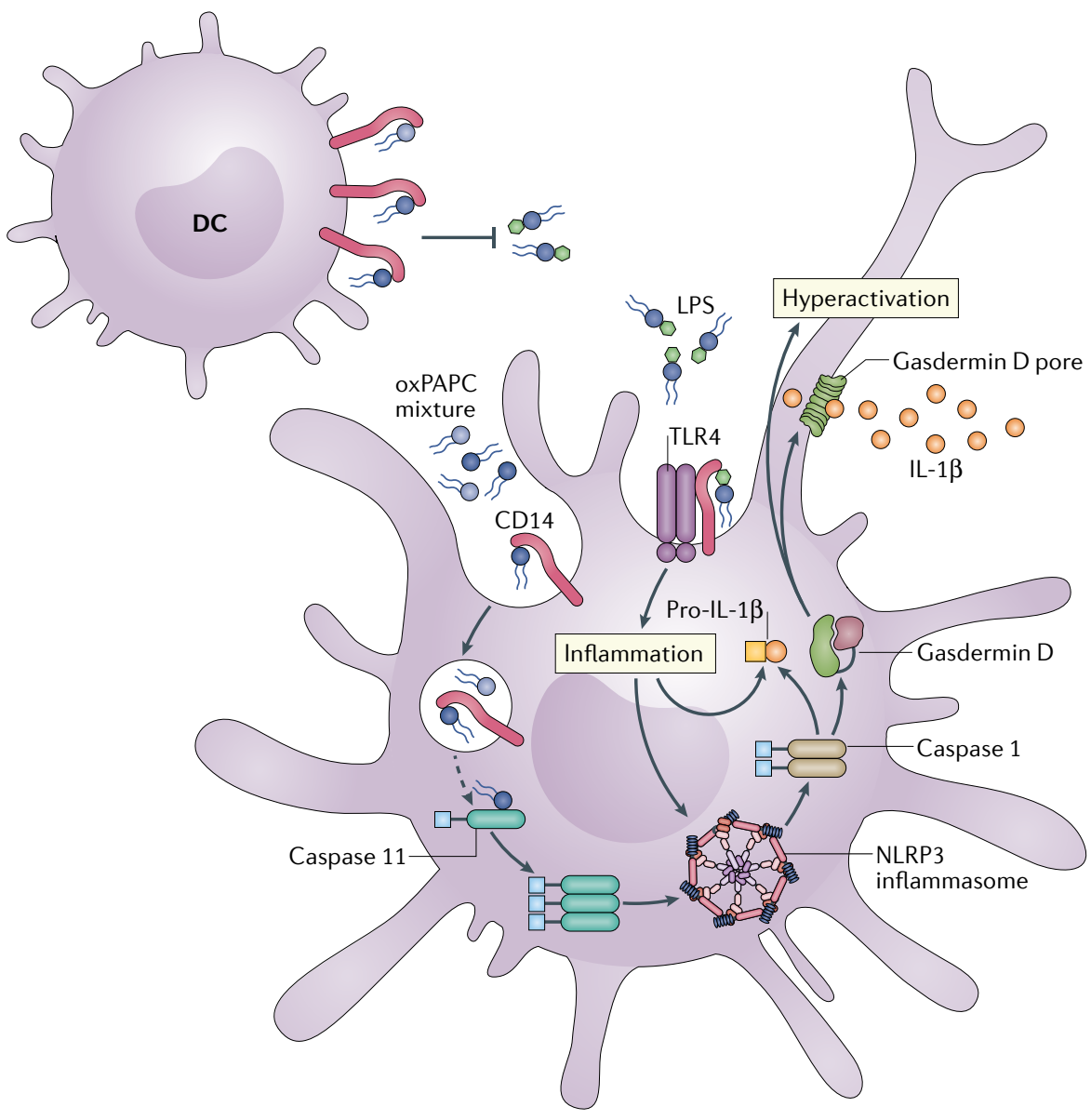

Fig. 2 | Mechanism of myeloid cell hyperactivation by oxidized phosphocholines. Lipopolysaccharide (LPS) is captured by surface CD14, which delivers this lipid to membrane-associated MD2 and Toll-like receptor 4 (TLR4). CD14 then transports LPS and TLR4 into the cell. TLR4 activation unleashes signalling pathways that stimulate an inflammatory response, resulting in the upregulation of many genes, including IL1B and NLRP3. This signal is recognized as a priming signal. Oxidized 1-palmitoyl-2-arachidonoyl-sn-glycero-3-phosphocholine derivatives (oxPAPCs) are also captured by CD14 and transported into the cell. Somehow oxPAPCs leave the endosomes and bind to caspase 11. This interaction leads to the oligomerization of an NLR family pyrin domain-containing protein 3 (NLRP3) inflammasome that results in a hyperactive state, in which caspase 1 cleaves pro-IL-1 $\beta$ into its mature form and gasdermin D (GSDMD) into its active form. Hyperactive dendritic cells (DCs) induce IL-1 $\beta$ release without the commitment to pyroptosis. As depicted in the cell at the top of the figure, oxPAPC binding to CD14 prevents subsequent interactions with LPS. 


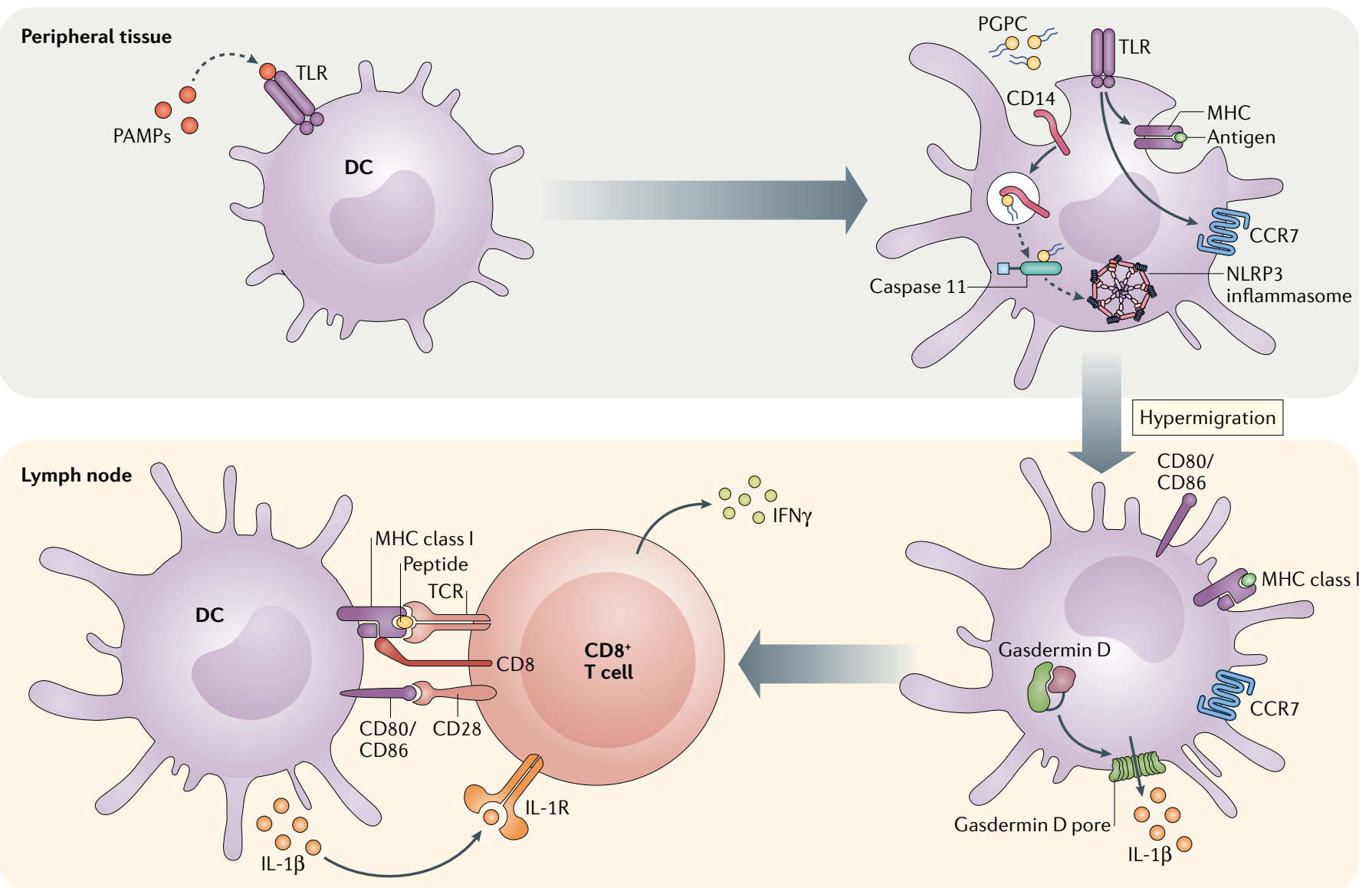

Fig. 3 | Hyperactive DCs control antitumour T cell immunity. Upon pathogen encounter, immature dendritic cells (DCs) circulating in the peripheral tissue recognize pathogen-associated molecular patterns (PAMPs) via Toll like-receptors (TLRs) and shift their activities from a naive state to an active state. In the presence of tissue damage, CD14 captures 1-palmitoyl-2-glutaroylsn-glycero-3-phosphocholine (PGPC) and delivers the lipids to caspase 11 to induce NLR family pyrin domain-containing protein 3 (NLRP3) activation.
These DCs achieve a long-lived state of hyperactivation, leading to IL-1 $\beta$ release from living DCs. Hyperactive DCs upregulate CC-chemokine receptor 7 (CCR7) expression and retain the ability for antigen uptake and processing of antigenic peptide on MHC class I. Hyperactive DCs carrying antigens then migrate to the draining lymph node via CCR7, where they activate CD $8^{+}$ T cells. IL-1 $\beta$ secreted by hyperactive DCs signal via IL-1 receptor (IL-1R) to stimulate T cell effector function. IFN $\gamma$, interferon- $\gamma$; TCR, T cell receptor. antitumour immunity, we return to the central thesis we have proposed. We propose that the conditions of DC hyperactivation where cells are exposed to generic indicators of microorganisms (TLR ligands) and tissue injury (oxPAPCs) - mimic the microenvironment of a virulent infection. Under these conditions, DCs receive multiple signals necessary to maximally enhance their migratory activities, metabolic activities and $\mathrm{T}$ cell stimulatory activities. The result of this enhancement is a superior adaptive immune response that enhances antitumour immunity. While the genesis of our logic derives from consideration of infectious encounters, these ideas have yet to be tested in this context. Of note, oxPLs, including oxidized low-density lipoprotein, are highly enriched in the tumour microenvironment. It was shown that oxidized low-density lipoprotein induces lipid peroxidation that suppresses $\mathrm{CD}^{+} \mathrm{T}$ cell effector functions in the tumour in a CD36-dependent manner ${ }^{57}$. These data provide the mandate to explore the links between lipid oxidation and cancer immunotherapy.

\section{Unrecognized sources of oxPAPCs may} underlie diverse immunotherapies. Although the role of hyperactive DCs in adaptive immunity needs further exploration, it is worth considering whether the protective activities of current therapies may depend on previously unrecognized oxPAPC sources that drive cell hyperactivation. For example, the chemotherapeutic chemicals oxaliplatin and anthracycline stimulate IL- $1 \beta$ production via NLRP3 inflammasomes, leading to tumour-specific $\mathrm{CD}^{+} \mathrm{T}$ cell responses ${ }^{57}$. Oxaliplatin is known to stimulate ROS, whose activities may generate PGPC and other oxPAPCs within the tumour. These factors would act with any coexisting PAMPs to hyperactivate DCs and prime antitumour $\mathrm{T}$ cell responses.
Consistent with this idea is evidence that patients with breast cancer bearing mutations in the inflammasome regulator P2X7R can develop rapid metastatic disease ${ }^{57,58}$. Total body irradiation should also induce oxPAPC formation, via the generation of ROS that occurs in these contexts. The impact of radiation-induced (or chemotherapy-induced) oxPAPC production on tumour immunity remains speculative. If these ideas are proven in humans, it is possible that vaccines could be engineered to specifically introduce oxPL moieties as adjuvants to promote more effective antigen-specific $\mathrm{T}$ cell responses.

\section{Conclusions and perspectives}

We suggest that heterogeneous oxPAPC mixtures or pure oxPAPC components cannot be classified as intrinsically proinflammatory or anti-inflammatory molecules. It is likely that the pleiotropic effects of oxPAPCs work in concert 
in vivo. In this regard, our knowledge is currently limited, as little is known about the identity and function of specific oxPAPC compounds. Further research is required to better understand the biological role of oxPAPCs during infectious diseases and other threats to the host. Recent evidence indicates that PGPC and POVPC can prevent vesicular stomatitis virus entry, thereby limiting viral replication ${ }^{59}$. Although the mechanism by which oxPAPCs inhibit viral entry is undetermined, these data provide the mandate to explore the interplay of pathogens and oxPLs, which may open new avenues for novel therapeutic targets.

Furthermore, a better understanding of how endogenous oxPLs impact phagocyte function is crucial for uncovering the impact of oxPLs on homeostasis and pathogenesis. POVPC abundance is increased in the lungs of aged mice, and these levels are further augmented upon inflammatory stimulation $^{60}$. In addition, the clearance of oxPLs from lungs was delayed in aged mice ${ }^{60}$, and correlated with severe lung injury and delayed resolution. In this regard, several questions remain unexplored. How do these oxPLs regulate phagocyte function in the ageing host? Can these lipids regulate the inflammaging process? This knowledge could support the development of effective vaccination strategies designed for elderly patients.

oxPLs enzymatically derived from innate immune cells were shown to promote coagulation factor function which restored thrombin generation. Therefore, it was proposed that bioactive oxPLs may be a target in bleeding and vascular inflammation disorders ${ }^{61,62}$. Interestingly, pyroptotic macrophages were shown to release tissue factor (an essential initiator of coagulation cascades) following inflammasome activation, which triggered blood clotting ${ }^{63}$. Whether oxPLs can regulate blood clotting via inflammasome activation is a possibility.

Finally, oxPLs are not the only stimuli that can induce a state of DC hyperactivation. Other hyperactivating stimuli besides oxPLs have been reported ${ }^{64}$, including bacterial peptidoglycan ${ }^{65}$, Staphylococcus aureus $\Delta$ oatA mutants ${ }^{66}$ and the TLR agonist R848 plus muramyl dipeptide $^{67}$. The latter induces human cDC2 hyperactivation that enhances $\mathrm{T}$ helper 1 and $\mathrm{T}$ helper 17 cell responses. Whether hyperactivating stimuli could be generally classified as boosters of adaptive T cell immunity or context-dependent regulators of immunity requires further investigation.
Dania Zhivaki ${ }^{1}$ and Jonathan C. Kagan (D) ${ }^{\otimes}$

Division of Gastroenterology, Boston Children's Hospital and Harvard Medical School, Boston, MA, USA.

凶e-mail: jonathan.kagan@childrens.harvard.edu https://doi.org/10.1038/s41577-021-00618-8

Published online 21 September 2021

1. Medzhitov, R. \& Janeway, C. A. Innate immunity: the virtues of a nonclonal system of recognition. Cell 91, 295-298 (1997).

2. Janeway, C. A. \& Medzhitov, R. Innate immune recognition. Annu. Rev. Immunol. 20, 197-216 (2002).

3. Pasare, C. $\&$ Medzhitov, R. Control of B-cell responses by Toll-like receptors. Nature 438, 364-368 (2005).

4. Iwasaki, A. \& Medzhitov, R. Control of adaptive immunity by the innate immune system. Nat. Immunol. 16, 343-353 (2015).

5. Fitzgerald, K. A. \& Kagan, J. C. Toll-like receptors and the control of immunity. Cell 180, 1044-1066 (2020).

6. Kawai, T. \& Akira, S. The role of pattern-recognition receptors in innate immunity: update on toll-like receptors. Nat. Immunol. 11, 373-384 (2010).

7. Vance, R. E. Immunology taught by bacteria. J. Clin. Immunol. 30, 507-511 (2010).

8. Miao, E. A. \& Warren, S. E. Innate immune detection of bacterial virulence factors via the NLRC4 inflammasome J. Clin. Immunol. 30, 502-506 (2010).

9. Vance, R. E., Isberg, R. R. \& Portnoy, D. A. Patterns of pathogenesis: discrimination of pathogenic and nonpathogenic microbes by the innate immune system. Cell Host Microbe 6, 10-21 (2009).

10. Lamkanfi, M. \& Dixit, V. M. Mechanisms and functions of inflammasomes. Cell 157, 1013-1022 (2014).

11. Franchi, L., Muñoz-Planillo, R. \& Nũñez, G. Sensing and reacting to microbes through the inflammasomes. Nat. Immunol. 13, 325-332 (2012).

12. Evavold, C. L. \& Kagan, J. C. Inflammasomes: threatassessment organelles of the innate immune system Immunity 51, 609-624 (2019).

13. Kieser, K. J. \& Kagan, J. C. Multi-receptor detection of individual bacterial products by the innate immune system. Nat. Rev. Immunol. 17, 376-390 (2017).

14. Imai, Y. et al. Identification of oxidative stress and Toll-like receptor 4 signaling as a key pathway of acute lung injury. Cell 133, 235-249 (2008).

15. Berliner, J. A. \& Watson, A. D. A role for oxidized phospholipids in atherosclerosis. N. Engl. J. Med. 353, 9-11 (2005).

16. Leitinger, N. Oxidized phospholipids as modulators of inflammation in atherosclerosis. Curr. Op. Lipidol. 14 421-430 (2003).

17. Bochkov, V. N. et al. Generation and biological activities of oxidized phospholipids. Antioxid. Redox Signal. 12, 1009-1059 (2010)

18. Febbraio, M., Hajjar, D. P. \& Silverstein, R. L. CD36: a class $B$ scavenger receptor involved in angiogenesis, atherosclerosis, inflammation, and lipid metabolism. J. Clin. Invest. 108, 785-791 (2001)

19. Kar, N. S., Ashraf, M. Z., Valiyaveettil, M. \& Podrez, E. A. Mapping and characterization of the binding site for specific oxidized phospholipids and oxidized low density lipoprotein of scavenger receptor CD36. J. Biol. Chem. 283, 8765-8771 (2008).

20. Boullier, A. et al. Phosphocholine as a pattern recognition ligand for CD36. J. Lipid Res. 46 969-976 (2005)

21. Zanoni, I., Tan, Y., di Gioia, M., Springstead, J. R. \& Kagan, J. C. By capturing inflammatory lipids released from dying cells, the receptor CD14 induces inflammasome-dependent phagocyte hyperactivation. Immunity 47, 697-709 e3 (2017).

22. Tan, Y., Zanoni, I., Cullen, T. W., Goodman, A. L. ¿ Kagan, J. C. Mechanisms of Toll-like receptor 4 endocytosis reveal a common immune-evasion strategy used by pathogenic and commensal bacteria. Immunity 43, 909-922 (2015).

23. Shirey, K. A. et al. The TLR4 antagonist eritoran protects mice from lethal influenza infection. Nature 497, 498-502 (2013).

24. Zanoni, I. et al. An endogenous caspase-11 ligand elicits interleukin-1 release from living dendritic cells. Science 352, 1232-1236 (2016).

25. Shi, J. et al. Inflammatory caspases are innate immune receptors for intracellular LPS. Nature 514, 187-192 (2014).
26. Kayagaki, N. et al. Noncanonical inflammasome activation by intracellular LPS independent of TLR4. Science 341, 1246-1249 (2013).

27. Dingjan, I. et al. Lipid peroxidation causes endosoma antigen release for cross-presentation. Sci. Rep. 6 , 22064 (2016).

28. Ashraf, M. Z. \& Srivastava, S. In Lipoproteins: Role in Health and Diseases (ed. Kostner, G.) Ch. 17 (IntechOpen, 2012).

29. Matt, U., Sharif, O., Martins, R. \& Knapp, S Accumulating evidence for a role of oxidized phospholipids in infectious diseases. Cell. Mol. Life SCi. 72, 1059-1071 (2015)

30. Bochkov, V. et al. Pleiotropic effects of oxidized phospholipids. Free Radic. Biol. Med. 111, 6-24 (2017).

31. Knapp, S., Matt, U., Leitinger, N. \& van der Poll, T. Oxidized phospholipids inhibit phagocytosis and impair outcome in gram-negative sepsis in vivo. J. Immunol. 178, 993-1001 (2007).

32. Thimmulappa, R. K. et al. Oxidized phospholipids impair pulmonary antibacterial defenses: evidence in mice exposed to cigarette smoke. Biochem. Biophys. Res. Commun. 426, 253-259 (2012)

33. Greenberg, M. E. et al. Oxidized phosphatidylserineCD36 interactions play an essential role in macrophage-dependent phagocytosis of apoptotic cells. J. Exp. Med. 203, 2613-2625 (2006).

34. Zhivaki, D. et al. Inflammasomes within hyperactive murine dendritic cells stimulate long-lived T cellmediated anti-tumor immunity. Cell Rep. 33, 108381 (2020).

35. Garlanda, C., Dinarello, C. A. \& Mantovani, A. The interleukin-1 family: back to the future. Immunity 39, 1003-1018 (2013)

36. Martinon, F., Burns, K. \& Tschopp, J. The inflammasome: a molecular platform triggering activation of inflammatory caspases and processing of prolL- $\beta$. Mol. Cell 10, 417-426 (2002)

37. Ding, J. et al. Pore-forming activity and structural autoinhibition of the gasdermin family. Nature $\mathbf{5 3 5}$ 111-116 (2016)

38. Heilig, R. et al. The gasdermin-D pore acts as a conduit for IL-1 $\beta$ secretion in mice. Eur. J. Immunol. 48, 584-592 (2018)

39. Evavold, C. L. et al. The pore-forming protein gasdermin D regulates interleukin-1 secretion from living macrophages. Immunity 48, 35-44 (2017).

40. Xia, S. et al. Gasdermin D pore structure reveals preferential release of mature interleukin-1. Nature 593, 607-611 (2021).

41. Rühl, S. et al. ESCRT-dependent membrane repair negatively regulates pyroptosis downstream of GSDMD activation. Science 362, 956-960 (2018).

42. di Gioia, M. et al. Endogenous oxidized phospholipids reprogram cellular metabolism and boost hyperinflammation. Nat. Immunol. 21, 42-53 (2020).

43. Di Gioia, M. \& Zanoni, I. Dooming phagocyte responses: inflammatory effects of endogenous oxidized phospholipids. Front. Endocrinol. https:/ doi.org/10.3389/fendo.2021.626842 (2021).

44. Serbulea, V. et al. Macrophages sensing oxidized DAMPs reprogram their metabolism to support redox homeostasis and inflammation through a TLR2-Sykceramide dependent mechanism. Mol. Metab. 7, 23-34 (2018).

45. Oskolkova, O V et al Oxidized phospholipids are more potent antagonists of lipopolysaccharide than inducers of inflammation. J. Immunol. 185, 7706-7712 (2010).

46. Bochkov, V. N. et al. Protective role of phospholipid oxidation products in endotoxin-induced tissue damage. Nature 419, 77-81 (2002)

47. Walton, K. A. et al. Specific phospholipid oxidation products inhibit ligand activation of Toll-like receptors 4 and 2. Arterioscler. Thromb. Vasc. Biol. 23, 1197-1203 (2003).

48. Erridge, C., Kennedy, S., Spickett, C. M. \& Webb, D. J. Oxidized phospholipid inhibition of Toll-like receptor (TLR) signaling is restricted to TLR2 and TLR4: roles for CD14, LPS-binding protein, and MD2 as targets for specificity of inhibition. J. Biol. Chem. 283, 24748-24759 (2008)

49. Chu, L. H. et al. The oxidized phospholipid oxPAPC protects from septic shock by targeting the non-canonical inflammasome in macrophages. Nat. Commun. 9, 996 (2018).

50. Bretscher, P. et al. Phospholipid oxidation generates potent anti-inflammatory lipid mediators that mimic structurally related pro-resolving eicosanoids by activating Nrf2. EMBO Mol. Med. 7, 593-607 (2015). 
51. Mendel, I. et al. A lecinoxoid, an oxidized phospholipid small molecule, constrains CNS autoimmune disease. J. Neuroimmunol. 226, 126-135 (2010)

52. Mendel, I., Yacov, N., Shoham, A., Ishai, E. \& Breitbart, E. Treatment with oxidized phospholipids directly inhibits nonalcoholic steatohepatitis and liver fibrosis without affecting steatosis. Dig. Dis. Sci. 61 , 2545-2553 (2016)

53. Jain, A., Song, R., Wakeland, E. K. \& Pasare, C. $T$ cell-intrinsic IL-1 R signaling licenses effector cytokine production by memory CD4 T cells. Nat. Commun. $\mathbf{9}$, $1-13$ (2018)

54. Ott, P. A. et al. An immunogenic personal neoantigen vaccine for patients with melanoma. Nature 547 217-221 (2017).

55. Castle, J. C. et al. Exploiting the mutanome for tumor vaccination. Cancer Res. 72, 1081-1091 (2012).

56. Hu, Z. et al. Personal neoantigen vaccines induce persistent memory $\mathrm{T}$ cell responses and epitope spreading in patients with melanoma. Nat. Med. 27, 515-525 (2021)

57. Ghiringhelli, F. et al. Activation of the NLRP3 inflammasome in dendritic cells induces IL-1Bdependent adaptive immunity against tumors. Nat. Med. 15, 1170-1178 (2009).

58. Casares, N. et al. Caspase-dependent immunogenicity of doxorubicin-induced tumor cell death. J. Exp. Med. 202, 1691-1701 (2005)

59. Ernandes, M. J. \& Kagan, J. C. Interferon-independent restriction of RNA virus entry and replication by a class of damage-associated molecular patterns. $\mathrm{mBio}$ 12, e00584-21 (2021).

60. Ke, Y. et al. Elevated truncated oxidized phospholipids as a factor exacerbating ALI in the aging lungs. FASEB J. 33, 3887-3900 (2019)

61. Slatter, D. A. et al. Enzymatically oxidized phospholipids restore thrombin generation in coagulation factor deficiencies. JCI Insight 3, e98459 (2018).

62. O'Donnell, V. B., Aldrovandi, N., Murphy, R. C. \& Krönke, G. Enzymatically oxidized phospholipids assume center stage as essential regulators of innate immunity and cell death. Sci. Signal. 12, eaau2293 (2019).

63. Wu, C. et al. Inflammasome activation triggers blood clotting and host death through pyroptosis. Immunity 50, 1401 (2019).

64. Zhivaki, D. \& Kagan, J. C. NLRP3 inflammasomes that induce antitumor immunity. Trends Immunol. 42, 575-589 (2021)

65. Wolf, A. J. et al. Hexokinase is an innate immune receptor for the detection of bacterial peptidoglycan. Cell 166, 624-636 (2016).

66. Shimada, T. et al. Staphylococcus aureus evades lysozyme-based peptidoglycan digestion that links phagocytosis, inflammasome activation, and IL-1 secretion. Cell Host Microbe 7, 38-49 (2010).

67. Hatscher, L. et al. Select hyperactivating NLRP3 ligands enhance the $\mathrm{TH} 1$ - and $\mathrm{TH} 17$-inducing potential of human type 2 conventional dendritic cells Sci. Signal. 14, eabe 1757 (2021).

68. Brubaker, S. W., Bonham, K. S., Zanoni, I. \& Kagan, J. C. Innate immune pattern recognition: a cell biological perspective. Annu. Rev. Immunol. 33, 257-290 (2015).

69. Matzinger, P. The danger model: a renewed sense of self. Science 296, 301-305 (2002).

70. Kagan, J. C. Lipopolysaccharide detection across the kingdoms of life. Trends Immunol. 38, 696-704 (2017).

71. Gauthier, A. E. et al. Deep-sea microbes as tools to refine the rules of innate immune pattern recognition. Sci. Immunol. 6, eabe0531 (2021).
72. Bianchi, M. E. DAMPs, PAMPs and alarmins: all we need to know about danger. J. Leukoc. Biol. 81, 1-5 (2007).

73. Lorent, J. H. et al. Plasma membranes are asymmetric in lipid unsaturation, packing and protein shape. Nat. Chem. Biol. 16, 644-652 (2020).

74. Bernhard, W. et al. Phosphatidylcholine molecular species in lung surfactant composition in relation to respiratory rate and lung development. Am. J. Resp. Cell Mol. Biol. 25, 725-731 (2001).

75. Bergmark, C. et al. A novel function of lipoprotein [a] as a preferential carrier of oxidized phospholipids in human plasma. J. Lipid Res. 49, 2230-2239 (2008).

76. O’Donnell, V. B., Rossjohn, J. \& Wakelam, M. J. O Phospholipid signaling in innate immune cells. J. Clin. Invest. 128, 2670-2679 (2018).

77. Hajeyah, A. A. Griffiths, W. J., Wang Y., Finch, A J \& O'Donnell, V. B. The biosynthesis of enzymatically oxidized lipids. Front. Endocrinol. https://doi.org/ 10.3389/fendo.2020.591819 (2020).

78. Huber, J. et al. Oxidized membrane vesicles and blebs from apoptotic cells contain biologically active oxidized phospholipids that induce monocyte-endothelial interactions. Arterioscler. Thromb. Vasc. Biol. 22 , 101-107 (2002)

79. Tsiantoulas, D. et al. Circulating microparticles carry oxidation-specific epitopes and are recognized by natural IgM antibodies. J. Lipid Res. 56, 440-448 (2015).

80. Watson, A. D. et al. Structural identification by mass spectrometry of oxidized phospholipids in minimally oxidized low density lipoprotein that induce monocyte/ endothelial interactions and evidence for their presence in vivo. J. Biol. Chem. 272, 13597-13607 (1997).

81. Qin, J., Goswami, R., Balabanov, R. \& Dawson, G. Oxidized phosphatidylcholine is a marker for neuroinflammation in multiple sclerosis brain J. Neurosci. Res. 85, 977-984 (2007).

82. Kanter, J. L. et al. Lipid microarrays identify key mediators of autoimmune brain inflammation. Nat. Med. 12, 138-143 (2006)

83. Sun, X. et al. Neutralization of oxidized phospholipids ameliorates non-alcoholic steatohepatitis. Cell Metab. 31, 189-206.e8 (2020).

84. Bochkov, V. N. et al. Oxidized phospholipids stimulate angiogenesis via autocrine mechanisms, implicating a novel role for lipid oxidation in the evolution of atherosclerotic lesions. Circ. Res. 99, 900-908 (2006).

85. Bochkov, V. et al. Novel immune assay for quantification of plasma protective capacity against oxidized phospholipids. Biomark. Med. 10, 797-810 (2016).

86. Gruber, F. et al. Photooxidation generates biologically active phospholipids that induce heme oxygenase- 1 in skin cells. J. Biol. Chem. 282, 16934-16941 (2007).

87. Que, X. et al. Oxidized phospholipids are proinflammatory and proatherogenic in hypercholesterolemic mice. Nature 558, 301 (2018).

88. Ståhle, M. et al. Therapeutic antibody against phosphorylcholine preserves coronary function and attenuates vascular ${ }^{18} \mathrm{~F}$-FDG uptake in atherosclerotic mice. JACC Basic Transl. Sci. 5, 360-373 (2020).

89. Inaba, K. et al. The formation of immunogenic major histocompatibility complex class II-peptide ligands in lysosomal compartments of dendritic cells is regulated by inflammatory stimuli. J. Exp. Med. 191, 927-936 (2000).

90. Mellman, I. \& Steinman, R. M. Dendritic cells: specialized and regulated antigen processing machines. Cell 106, 255-258 (2001)

91. Joffre, O., Nolte, M. A., Spörri, R. \& Reis e Sousa, C. Inflammatory signals in dendritic cell activation and the induction of adaptive immunity. Immunol. Rev. 227, 234-247 (2009).

92. Sallusto, F. \& Lanzavecchia, A. The instructive role of dendritic cells on T-cell responses. Arthr. Res. 4 S127-S132 (2002).

93. Sabado, R. L., Balan, S. \& Bhardwaj, N. Dendritic cell-based immunotherapy. Cell Res. 27, 74-95 (2017).

94. Wculek, S. K. et al. Dendritic cells in cancer immunology and immunotherapy. Nat. Rev. Immunol. 20, 7-24 (2020).

95. Draube, A. et al. Dendritic cell based tumor vaccination in prostate and renal cell cancer: a systematic review and meta-analysis. PLOS ONE 6, e18801 (2011).

96. Hu, Z., Ott, P. A. \& Wu, C. J. Towards personalized, tumour-specific, therapeutic vaccines for cancer. Nat. Rev. Immunol. 18, 168-182 (2017).

97. Ben-Sasson, S. Z. et al. IL-1 acts directly on CD4 $T$ cells to enhance their antigen-driven expansion and differentiation. Proc. Natl Acad. Sci. USA 106, 7119-7124 (2009).

98. Ben-Sasson, S. Z et al. IL-1 enhances expansion, effector function, tissue localization, and memory response of antigen-specific CD8 T cells. J. Exp. Med. 210, 491-502 (2013).

99. Lu, A. et al. Unified polymerization mechanism for the assembly of ASC-dependent inflammasomes. Cell 156, 1193-1206 (2014).

100. Kagan, J. C., Magupalli, V. G. \& Wu, H. SMOCs supramolecular organizing centres that control innate immunity. Nat. Rev. Immunol. 14, 821-826 (2014).

101. Mempel, T. R., Henrickson, S. E. \& von Andrian, U. H. T-cell priming by dendritic cells in lymph nodes occurs in three distinct phases. Nature 427, 154-159 (2004).

102. Eisenbarth, S. C. et al. Crucial role for the Nalp3 inflammasome in the immunostimulatory properties of aluminium adjuvants. Nature 453, 1122-1126 (2008).

103. Kool, M. et al. Cutting edge: alum adjuvant stimulates inflammatory dendritic cells through activation of the NALP3 inflammasome. J. Immunol. 181, 3755-3759 (2008).

104. Marrack, P., McKee, A. S. \& Munks, M. W. Towards an understanding of the adjuvant action of aluminium Nat. Rev. Immunol 9, 287-293 (2009).

\section{Acknowledgements}

The authors thank the members of the Kagan laboratory for helpful discussions. This work was supported by US National Institutes of Health grants AI 133524, Al093589, Al116550 and P30DK34854 (J.C.K.). D.Z. was funded by the Charles King Trust Fellowship.

\section{Author contributions}

The authors contributed equally to all aspects of the article.

\section{Competing interests}

J.C.K. holds equity in and consults for IFM Therapeutics and Corner Therapeutics. D.Z. holds equity in and consults for Corner Therapeutics. None of these relationships influenced the manuscript.

Peer review information

Nature Reviews Immunology thanks V. Bochkov and the other, anonymous, reviewer(s) for their contribution to the peer review of this work.

\section{Publisher's note}

Springer Nature remains neutral with regard to jurisdictional claims in published maps and institutional affiliations.

(c) Springer Nature Limited 2021 